\title{
Head and Neck Cancers: Advantages of Advanced Radiation Therapy and Importance of Supportive Care
}

\author{
Presented by Sharon Spencer, MD
}

\section{Abstract}

For more than a decade, the incidence of head and neck cancers has been increasing in the United States. Worldwide, they are the ninth most common cancer. Today, newer advances in radiotherapy (RT), such as fractionation, 4-dimensional cone-beam CT, and intensitymodulated RT, have provided clinicians with the opportunity for improved patient outcomes. At the NCCN 23rd Annual Conference, Sharon Spencer, MD, described the advantages of advances in RT and the means to mitigate untoward side effects.

J Natl Compr Canc Netw 2018;16(5.5):666-669 doi: 10.6004/jncen.2018.0049

According to Sharon Spencer, MD, Professor and Chief of Medical Services, Department of Radiation Oncology, University of Alabama at Birmingham Comprehensive Cancer Center and Vice Chair of the NCCN Guidelines Panel for Head and Neck (H\&N) Cancers, there may be a bright future ahead for patients with $H \& N$ cancers and for the clinicians who treat them. Advances in radiation therapy (RT) have given Dr. Spencer and her colleagues some formidable tools to manage their patients with $H \& N$ cancers. "As we look at outcomes," she noted at the NCCN 23rd Annual Conference, "we are seeing improvement. But we can do better."

$\mathrm{H} \& \mathrm{~N}$ squamous cell carcinoma (HNSCC) is a heterogeneous disease, encompassing a variety of tumors that originate in the hypopharynx, oropharynx, lip, oral cavity, nasopharynx, or larynx. $H \& N$ cancers are the ninth most common cancer worldwide, with 350,000 new cases diagnosed yearly. ${ }^{1}$ Oral cancers have an $80 \%$ to $90 \%$ survival rate when found at early stages. However, most patients present with stage III and IV locally advanced H\&N cancers. "Unfortunately," emphasized

Presented by Sharon Spencer, MD, Department of Radiation Oncology, University of Alabama at Birmingham Comprehensive Cancer Center, Birmingham, Alabama.

Dr. Spencer has disclosed that she has no financial interests, arrangements, affiliations, or commercial interests with the manufacturers of any products discussed in this article or their competitors.

Correspondence: Sharon Spencer, MD, Department of Radiation Oncology, University of Alabama at Birmingham Comprehensive Cancer Center, 1700 6th Avenue South, Birmingham, AL 35233.

Email: sspencer@uabmc.edu
Dr. Spencer, "this is the reason for the very high 5 -year death rate of $43 \%$."

Today, patients with locally advanced HNSCC require multimodality treatment. This diverse population is ideally best managed by a comprehensive multidisciplinary team. Sometimes trimodality therapy consisting of surgery, RT, and chemotherapy is required. In the setting of organ preservation, combination chemoradiotherapy (CRT) is appropriate.

\section{Human Papillomavirus and HNSCC Cancers}

Human papillomavirus (HPV)-driven oropharyngeal squamous cell carcinomas represent a distinct disease from other HNSCCs, which are traditionally induced by excessive tobacco and alcohol consumption. ${ }^{2}$ According to Dr. Spencer, one of the most striking clinical features of HPV-related oropharyngeal squamous cell carcinomas is their very good prognosis, with the risk of death among HPV-positive patients being half that of their HPV-negative counterparts. ${ }^{3}$

The phase II ECOG-ACRIN 1308 trial was an attempt to deintensify therapy in the HPV population that typically has an excellent outcome with current standard therapies. Patients underwent induction chemotherapy followed by clinical and radiographic reevaluations. Responding patients were then treated with weekly cetuximab and reduced-dosed RT at 54 Gy. Patient outcomes were encouraging, as significantly fewer patients treated with the 54 Gy of RT experienced dif- 
ficulty swallowing solids. There is promise that longterm toxicity decrements will be seen. ${ }^{4}$

Results from the phase II OPTIMA trial indicated that HPV-positive patients with oropharynx cancer may potentially receive lower doses of RT. ${ }^{5}$ Patients received induction carboplatin and nabpaclitaxel followed by reevaluation. Those who were considered low risk and experienced a response of $\geq 50 \%$ received RT alone at 50 Gy. For patients who were considered low risk and had a 30\% to $50 \%$ response, or who were considered high risk and achieved a response of $\geq 50 \%$ received 45 Gy and CRT. All other cases received what was considered standard doses of CRT. All patients received volume reductions in RT fields limited to the first echelon of uninvolved lymph nodes. The study investigators concluded that response to induction chemotherapy is a useful biomarker for dose and volume reductions.

Other trials have closed that were also designed to decrease toxicity by modifying standard treatment strategies. For example, the closed NRG-HN002 was designed to attempt to deintensify therapy in HPVpositive patients who had a limited smoking history. This phase II trial randomized patients to receive a total of 60 Gy of RT given over 5 weeks versus 60 Gy of RT given over 6 weeks along with weekly cisplatin at $40 \mathrm{mg} / \mathrm{m} 2$. This trial will hopefully provide some useful information.

\section{Overview of RT Techniques}

"Accurate positioning in the radiation treatment of patients with H\&N cancer is important, since multiple critical organs surround the target," noted Dr. Spencer (Figure 1). "Setup errors can result in significant underdosing to the tumor and/or overdosing to $\geq 1$ critical organs. In theory, 3D imaging methods are expected to better visualize internal landmarks and can lead to more accurate tumor and normal tissue definition, as well as more accurate daily setups" (Figure 2). In one study, the discrepancy between $2 \mathrm{D} / 3 \mathrm{D}$ and $3 \mathrm{D} / 3 \mathrm{D}$ image registration was found to increase slightly from cranial to $\mathrm{C}$-spine to $\mathrm{T}$-spine to L-spine sites. ${ }^{6}$

Another technique discussed was volumetric modulated arc therapy. This is a novel radiation technique that can achieve highly conformal dose distributions with improved target volume coverage and sparing of normal tissues.

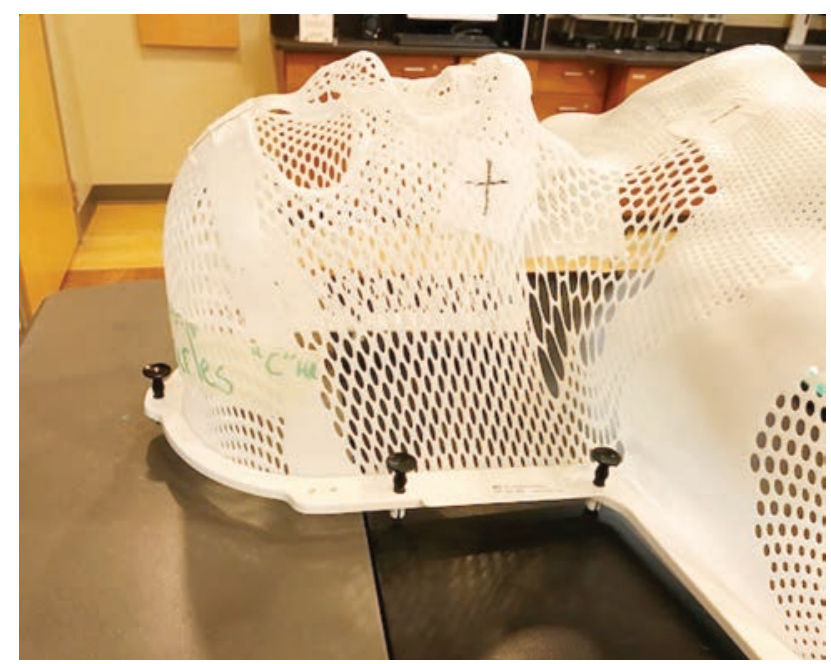

Figure 1. Mask for radiotherapy.

Four-dimensional cone-beam CT is another technique that uses a constant gantry speed and imaging frequency that are independent of the patient's breathing rate. It has been demonstrated that by varying the gantry speed and imaging frequency in response to changes in the patient's real-time respiratory signal, the imaging dose can be reduced by $50 \%$ to $70 \%$. $^{7}$

IMRT has become an important radiation-delivery technique in the management of $H \& N$ cancers. It conforms the prescribed doses to the target volumes of complex shapes while sparing the adjacent critical structures without compromising the target coverage. "Moreover," explained Dr. Spencer, "IMRT can enhance the fluence at the margins of the target and compensate for the beam penumbra without extending the portal boundaries." Another distinct advantage of IMRT is that it makes it possible to deliver different doses to different target volumes in a single plan, commonly referred to as simultaneous integrated boost IMRT. ${ }^{8}$

\section{IMRT: Preferred Technique}

"RT has benefited from advances in cancer imaging, treatment planning computer software, and developments in radiation delivery technology. It is now one of the most technology-driven branches of medicine," stressed Dr. Spencer. The preferred technique for patients with H\&N cancers is IMRT, the main advantage of which is to confine the higher radia- 


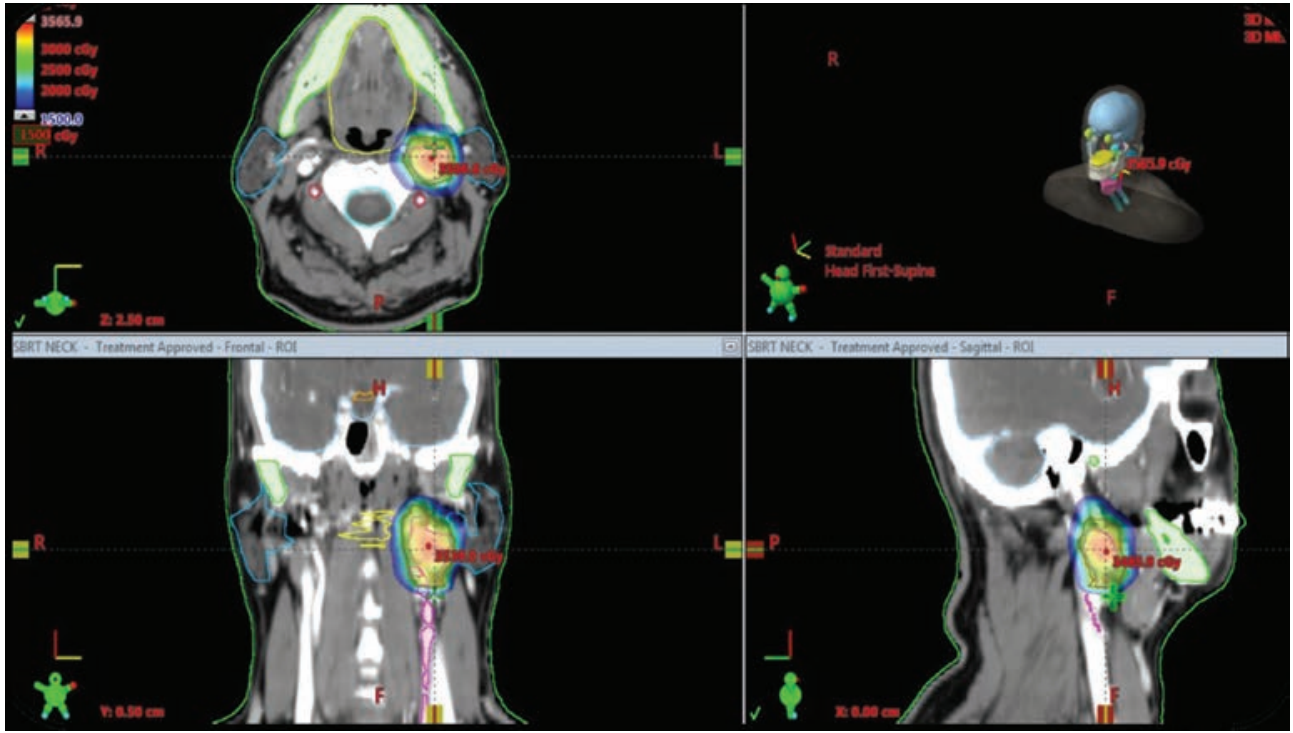

Figure 2. Four-fold view of head and neck imaging.

tion doses to the target volumes and, therefore, offer better protection of the surrounding organs at risk.

The PARSPORT phase III trial represents a convincing example to highlight improvements in IMRT over conventional RT. ${ }^{9}$ In IMRT treatment planning, whenever feasible, several volumetric constraints should be used. The total dose of radiation ranges from 50 to $70 \mathrm{~Gy}$, depending on tumor type and target volumes. "Unfortunately," stated Dr. Spencer, "although these high-energy beams are targeted to the tumor site as precisely as possible, there are intermediate and low doses, which can interact with normal tissues." Ongoing studies are exploring particle therapy using protons and heavy particle ions in attempts to minimize dose to normal tissues.

\section{What About Fractionation?}

The basis of fractionation is rooted in 5 primary biologic factors called the 5 Rs of RT: radiosensitivity, repair, repopulation, redistribution, and reoxygenation. Mammalian cells have different radiosensitivities and can repair radiation damage; this is a complex process that involves repair of sublethal damage by a variety of repair enzymes and pathways. Next, cells receiving fractionated doses of radiation can repopulate. The redistribution of the proliferating cell population throughout the cell cycle phases increases the cell kill from a fractionated treatment relative to a single session treatment. Lastly, reoxygenation of hypoxic cells occurs during a fractionated course of treatment, making them more radiosensitive to subsequent doses of radiation.

To effectively eliminate tumor cells and minimize side effects to normal tissue, conventional RT regimens deliver the prescribed radiation dose in multiple daily fractions (usually 2 Gy/fraction), given over several weeks. "To assure adequate target volume coverage and minimize the risk of RTinduced toxicity, an accurate definition of the organs at risk in the treatment plan is paramount," emphasized Dr. Spencer. Studies have confirmed that altered fractionation RT is associated with improved overall and progression-free survival when compared with conventional RT. ${ }^{10}$

To demonstrate the benefit of altered radiation fractionation schemes, Dr. Spencer provided evidence from the RTOG 90-03 trial. This study was designed to test whether altered radiation fractionation schemes versus standard fractionation improved local-regional control rates in patients with HNSCC. ${ }^{11}$ It was found that hyperfractionation and accelerated fractionation with concomitant boost decreased 5-year local-regional failure by $19 \%$ when compared with standard fractionation. "And hyperfractionation did so without increasing late toxicities," added Dr. Spencer.

The current standard fractionation is based on 5 daily treatments per week and a total treatment time of several weeks. This regimen reflects the practical 
aspects of dose delivery to a patient, successful outcome of patient treatments, and convenience to the staff delivering the treatment.

\section{Supportive Care}

All professionals caring for patients with $\mathrm{H} \& \mathrm{~N}$ cancer should assess supportive care needs in initial treatment planning and throughout the disease course. Support provided will need to accommodate any communication impediment.

Because treatments for most patients with $\mathrm{H} \& \mathrm{~N}$ cancers are likely to affect speech and swallowing, it is important to establish protocols within the multidisciplinary team to provide posttreatment support. ${ }^{12}$ Instrumental assessments of swallowing include flexible endoscopic examination of swallowing, videofluoroscopy, and/or modified barium swallow. Instrumental assessments of voice include endoscopy, stroboscopy, and speech studio/laryngography.

\section{Symptom Management}

Dr. Spencer discussed a number of side effects associated with RT in patients with H\&N cancers. "The majority of patients undergoing RT for H\&N cancers will develop oral mucositis," she stated. "Pain is the main debilitating symptom of mucositis." She noted that topical anesthetics, narcotics, and antidepressants could be used to control mucositis in these patients. Long-acting opioid patches may reduce the need for immediate-release opiates and may provide lengthier freedom from pain. "Oral mucositis usually appears early on in a radiotherapy regimen and is often the first acute side effect," Dr. Spencer said.

Another side effect associated with the treatment of H\&N cancers is xerostomia. Objectively, patients affected by xerostomia have a decrease of the salivary output, leading to functional oral disorders such as sore throat, altered taste, dental decay, changes in voice quality, and impaired chewing and swallowing. ${ }^{13}$ It may be managed with amifostine, pilocarpine, and cevimeline.

Management of trismus depends on the underlying cause and usually needs to be implemented in a timely fashion, before the condition worsens. "Mild cases of pain and dysfunction can be managed with heat therapy, analgesics, muscle relaxants, and a soft diet," noted Dr. Spencer.

Lastly, a combination side effect addressed by Dr. Spencer is odynophagia and dysphagia, which can occur as separate entities but most often occur together. Typically, the management of the symptoms is very similar. It is important, however, to ensure that the speech and swallowing professionals interact early and frequently with these patients.

\section{References}

1. Fitzmaurice C, Allen C, Barber RM, et al. Global, regional, and national cancer incidence, mortality, years of life lost, years lived with disability, and disability-adjusted life-years for 32 cancer groups, 1990 to 2015: a systematic analysis for the global burden of disease study. JAMA Oncol 2017;3:524-548.

2. Bouvard V, Baan R, Straif K, et al. WHO international agency for research on cancer monograph working group. A review of human carcinogens-part B: biological agents. Lancet Oncol 2009;10:321-322.

3. De Felice F, Polimeni A, Valentini V, et al. Radiotherapy controversies and prospective in head and neck cancer: a literature-based critical review. Neoplasia 2018;20:227-232.

4. Marur S, Li S, Cmelak AJ, et al. E1308: phase II trial of induction chemotherapy followed by reduced dose radiation and weekly cetuximab in patients with HPV associated respectable squamous cell carcinoma of the oropharynx-ECOG-ACRIN Cancer Research Group. J Clin Oncol 2016;35:490-497.

5. Melotek J, Seiwert TY, Blair EA, et al. Optima: a phase II dose and volume de-escalation trial for high- and low-risk HPV+ oropharynx cancers [abstract]. J Clin Oncol 2017;35(Suppl):Abstract 6066.

6. Li G, Yang TJ, Furtado H, et al. Clinical assessment of 2D/3D registration accuracy in 4 major anatomic sites using on-board 2D kilovoltage images for 6D patient setup. Technol Cancer Res Treat 2015;14:305-314.

7. O'Brien RT, Stankovic U, Sonke JJ, et al. Reducing 4DCBCT imaging time and dose: the first implementation of variable gantry speed 4DCBCT on a linear accelerator. Phys Med Biol 2017;62:4300-4317.

8. Jothybasu KS, Bahl A, Subramani V, et al. Static versus dynamic intensitymodulated radiotherapy: profile of integral dose in carcinoma of the nasopharynx. J Med Phys 2009;34:66-72.

9. Nutting CM, Morden JP, Harrington KJ, et al. Parotid-sparing intensity modulated versus conventional radiotherapy in head and neck cancer (PARSPORT): a phase 3 multicentre randomised controlled trial. Lancet Oncol 2011;12:127-136.

10. Lacas B, Bourhis J, Overgaard J, et al. Role of radiotherapy fractionation in head and neck cancers (MARCH): an updated meta-analysis. Lancet Oncol 2017;18:1221-1237.

11. Beitler JJ, Zhang Q, Fu KK, et al. Final results of local-regional control and late toxicity of RTOG 90-03: a randomized trial of altered fractionation radiation for locally advanced head and neck cancer. Int J Radiat Oncol Biol Phys 2014;89:13-20.

12. Clarke P, Radford $K$, Coffey $M$, et al. Speech and swallow rehabilitation in head and neck cancer. J Laryngol Otol 2016;130(Suppl 2):S176-180.

13. Pinna R, Campus G, Cumbo E, et al. Xerostomia induced by radiotherapy: an overview of the physiopathology, clinical evidence, and management of the oral damage. Ther Clin Risk Manag 2015;11:171-188. 